\title{
Retinoic acid ameliorates photoaged skin through RAR-mediated pathway in mice
}

\author{
ZHANGJUN LI, XINWU NIU, SHENGXIANG XIAO and HUIQUN MA
}

\begin{abstract}
Department of Dermatology, Second Affiliated Hospital of Xi'an Jiaotong University, Xi'an, Shaanxi 710004, P.R. China
\end{abstract}
Received March 10, 2017; Accepted August 14, 2017

DOI: $10.3892 / \mathrm{mmr} .2017 .7336$

\begin{abstract}
Retinoic acid (RA), the bioactive metabolite of vitamin A, has demonstrated efficacy in the treatment of photoaged skin; however, the mechanism of action of RA remains unclear. The aim of the present study was to examine whether the therapeutic effects of RA on photoaged skin are mediated by retinoic acid receptor (RAR) and/or retinoid $\mathrm{X}$ receptor ( $\mathrm{RXR}$ ) in mice, and to investigate the underlying mechanism. Photoaged skin in Imprinting Control Region mice was induced by repeated exposure to ultraviolet (UV) irradiation. Mice were randomly divided into nine groups: Normal; UV control; all-trans retinoic acid (ATRA); ATRA + RAR antagonist; ATRA + RXR antagonist; RAR agonist; RAR agonist + RAR antagonist; RXR agonist; and RXR agonist + RXR antagonist. Masson's trichrome staining was used to examine skin collagen fibers. Hydroxyproline assays were used to determine collagen content. The protein expression of matrix metalloproteinase (MMP)-3, MMP-13, type I procollagen, c-Jun and c-Fos was detected using western blot analysis. The results demonstrated that ATRA and RAR agonist ameliorated the UV-induced damage to skin collagen fibers, and increased the collagen content in photoaged skin through RAR. Furthermore, ATRA and RAR agonist stimulated type I procollagen protein expression, and inhibited MMP-3, MMP-13 and c-Jun protein expression through RAR in photoaged skin. However, ATRA and RAR agonist exhibited no significant effect on the protein expression of c-Fos in photoaged skin. These findings suggest that RA ameliorates photoaged skin through a RAR-mediated signaling pathway in mice.
\end{abstract}

Correspondence to: Professor Xinwu Niu, Department of Dermatology, Second Affiliated Hospital of Xi'an Jiaotong University, 157 Xiwu Road, Xi'an, Shaanxi 710004, P.R. China E-mail: nxw192@126.com

Abbreviations: AP-1, activator protein-1; ATRA, all-trans retinoic acid; Hyp, hydroxyproline; MED, minimal erythema dose; MMP, matrix metalloproteinase; RA, retinoic acid; RAR, retinoic acid receptor; $\mathrm{RXR}$, retinoid $\mathrm{X}$ receptor; $\mathrm{UV}$, ultraviolet

Key words: matrix metalloproteinases, photoaged skin, retinoic acid, retinoic acid receptor, retinoid X receptor, type I procollagen, ultraviolet irradiation

\section{Introduction}

Photoaging refers to premature skin aging caused by chronic exposure to ultraviolet (UV) irradiation, especially the UVA (315-400 nm) and UVB (280-315 nm) components, which are regarded as the main cause of skin damage $(1,2)$. Photoaged skin is characterized by coarse wrinkles, dryness, laxity, dyspigmentation, telangiectasia, a leathery appearance and histological changes, including variable epidermal thickness, solar elastosis and disorganization of collagen fibers $(1,3)$.

UV irradiation induces the expression of transcription factor activator protein-1 (AP-1), which plays an important role in the mechanism of photoaging (4). AP-1, mainly composed of c-Jun and c-Fos proteins, increases matrix metalloproteinases (MMPs) transcription and decreases procollagen synthesis (3). MMPs are a large family of zinc-containing endopeptidases that are responsible for the degradation of collagen and other extracellular matrix (ECM) proteins (5). Among them, MMP-1 (interstitial collagenase) initiates the cleavage of types I and III fibrillar collagen in human skin, while MMP-3 (stromelysin-1) activates proMMP-1 and further degrades the collagen fragments $(1,6)$. Rodents lack the MMP-1 gene, which is functionally replaced by the MMP-13 (collagenase-3) gene $(5,7)$. Therefore, MMP-3 and MMP-13 are the primary UV-induced collagenolytic enzymes in mouse skin. Type I procollagen is synthesized by dermal fibroblasts and subsequently converted into type I collagen, which is the major structural protein in dermal ECM. In the process of photoaging, UV irradiation decreases type I procollagen synthesis, resulting in the loss of collagen content (3). Based on the underlying mechanism of photoaging, the regulation of MMPs and type I procollagen may be an effective strategy for the prevention and treatment of photoaging.

Retinoic acid (RA), the bioactive metabolite of vitamin A, plays a key role in regulating proliferation and differentiation of cutaneous cells (8). It has been widely used in the treatment of dermatological disorders, such as acne, psoriasis, skin carcinoma and photoaging (9-12). In particular, all-trans retinoic acid (ATRA) is considered the gold standard to treat photoaged skin (13). Topical ATRA could improve several clinical and histological signs of photoaged skin, including improved skin appearance, increased anchoring fibrils and increased dermal collagen $(8,12-14)$. Classically, the actions of RA are mediated by retinoid nuclear receptors, retinoic acid receptor (RAR) and retinoid X receptor (RXR) (15). 
However, whether RA exerts its therapeutic effects on photoaged skin through retinoid nuclear receptors has not yet been elucidated. Therefore, the present study aimed to explore whether the therapeutic effects of RA on photoaged skin are mediated by RAR and/or RXR in mice and to investigate the underlying mechanism by histological examination of collagen fibers, determination of collagen content and detection of MMP-3, MMP-13, type I procollagen, c-Jun and c-Fos protein expression in mouse skin.

\section{Materials and methods}

Animals. Male ICR mice ( 8 weeks old) were obtained from the Laboratory Animal Center of Xi'an Jiaotong University (Xi'an, China). The animals were housed under controlled temperature $\left(23 \pm 2^{\circ} \mathrm{C}\right)$, humidity $(55 \pm 5 \%)$ and light $(12-\mathrm{h}$ light/dark cycle) with free access to standard diet and water. After acclimatization for 1 week, 72 mice were randomly allocated into two groups: non-irradiated group $(n=8)$ and UV-irradiated group $(n=64)$. The dorsal skin of mice $\left(2 \times 3 \mathrm{~cm}^{2}\right)$ was shaved using an electric razor, and this operation was repeated before UV irradiation and postirradiation treatment. All animal experiments were approved by the Laboratory Animal Administration Committee of $\mathrm{Xi}$ 'an Jiaotong University and performed according to the Guidelines for Animal Experimentation of Xi'an Jiaotong University and the Guide for the Care and Use of Laboratory Animals published by the US National Institutes of Health (NIH publication no. 85-23, revised 2011).

UV irradiation. UV irradiation of mice was performed using the UV light source provided by 2 UVA lamps (315-400 nm, peak wavelength: $365 \mathrm{~nm})$ and 4 UVB lamps (280-315 nm, peak wavelength: $312 \mathrm{~nm}$ ) (both from Beijing Lighting Research Institute, Beijing, China). The distance from the lamps to the animals' backs was $30 \mathrm{~cm}$. The minimal erythema dose (MED) was preliminarily measured with a UV meter (Lutron UV-340A, Taipei, Taiwan), and $1,200 \mathrm{~mJ} / \mathrm{cm}^{2}$ of UVA and $180 \mathrm{~mJ} / \mathrm{cm}^{2}$ of UVB were assembled $1 \mathrm{MED}$ in this study. Mice were irradiated 3 times a week (Monday, Wednesday and Friday) for 12 weeks. The irradiation dose was increased weekly by 1 MED from 1 MED up to 4 MED and then maintained at 4 MED for the rest weeks. The non-irradiated group was treated identically with the lamps power off.

Postirradiation treatment. After 12 weeks of UV irradiation, mice in the UV-irradiated group were randomly reallocated into eight groups with 8 mice per group: UV-irradiated plus vehicle (ethanol: propylene glycol, 7:3 v/v)-treated group (UV control group), UV-irradiated plus ATRA (Sigma-Aldrich, St. Louis, MO, USA)-treated group (ATRA group), UV-irradiated plus ATRA and AGN193109 (Santa Cruz Biotechnology, Inc., Santa Cruz, CA, USA)-treated group (ATRA + RAR antagonist group), UV-irradiated plus ATRA and HX531 (Tocris Bioscience, Bristol, UK)-treated group (ATRA + RXR antagonist group), UV-irradiated plus TTNPB (Sigma-Aldrich)-treated group (RAR agonist group), UV-irradiated plus TTNPB and AGN193109-treated group (RAR agonist + RAR antagonist group), UV-irradiated plus SR11237 (Sigma-Aldrich)-treated group (RXR agonist group) and UV-irradiated plus SR11237 and HX531-treated group (RXR agonist + RXR antagonist group). ATRA and retinoid receptor-specific agonists and antagonists were applied topically 5 times a week in $100 \mu \mathrm{l}$ vehicle per treatment for 8 weeks. According to previous studies (16-19), these agonists and antagonists were applied at the following concentrations with slight modification: ATRA, $160 \mathrm{nM}$; TTNPB, $160 \mathrm{nM}$; AGN193109, 400 nM; SR11237, 160 nM; HX531, 400 nM. Eight mice in the non-irradiated group were used as the normal group and treated with vehicle alone.

Histological examination. Twenty-four hour after the final treatment, mice were sacrificed by cervical dislocation under anesthesia, and dorsal skin was quickly removed. The skin samples were fixed in $10 \%$ neutral buffered formalin for $24 \mathrm{~h}$, embedded in paraffin and sectioned at $5 \mu \mathrm{m}$. Masson's trichrome staining was performed to examine the skin collagen fibers. The stained sections were captured under an Olympus BX51 light microscope equipped with a DP70 digital camera (Olympus, Tokyo, Japan).

Determination of collagen content. Hydroxyproline (Hyp) can be converted to the equivalent of collagen by multiplying the factor 7.46, considering Hyp is the almost exclusive amino acid of collagen and accounts for $13.4 \pm 0.24 \%$ of mammalian collagen in previous studies $(20,21)$. Hence, in this study, total Hyp content in the skin was measured using the Hyp assay kit (Nanjing Jiancheng Bioengineering Institute, Nanjing, China) according to the manufacturer's instruction for the determination of collagen content.

Western blot analysis. Fresh skin samples were homogenized in ice-cold RIPA lysis buffer (Heart Biological Technology, Co., Ltd., Xi'an, China). Lysates were centrifuged at 14,000 x g for $10 \mathrm{~min}$ at $4^{\circ} \mathrm{C}$, and the supernatants were collected as the total proteins. Protein concentration was measured using the BCA protein assay kit (Beyotime Institute of Biotechnology, Shanghai, China). Each sample was subsequently denatured by boiling in Laemmli loading buffer for 5 min. Equal amounts of protein were separated by $8-10 \%$ sodium dodecyl sulfate-polyacrylamide gel electrophoresis (SDS-PAGE) and transferred to PVDF membranes (Millipore, Billerica, MA, USA). After blocking with $5 \%$ non-fat dried milk for $2 \mathrm{~h}$ at $37^{\circ} \mathrm{C}$, the membranes were incubated overnight at $4^{\circ} \mathrm{C}$ with the following primary antibodies: rabbit anti-MMP-3 monoclonal antibody (Cat. no. ab52915; 1:1,000 dilution), rabbit anti-MMP-13 polyclonal antibody (Cat. no. ab39012; 1:1,000 dilution) (both from Abcam, Cambridge, MA, USA), rabbit anti-c-Jun monoclonal antibody (Cat. no. 9165; 1:1,000 dilution), rabbit anti-c-Fos monoclonal antibody (Cat. no. 2250; 1:1,000 dilution) (both from Cell Signaling Technology, Inc., Danvers, MA, USA), goat anti-type I procollagen polyclonal antibody (Cat. no. sc-8787; 1:500 dilution) and mouse anti- $\beta$-actin monoclonal antibody (Cat. no. sc-47,778; 1:1,000 dilution) (both from Santa Cruz Biotechnology, Inc.). After washing 3 times, the membranes were incubated with the appropriate horseradish peroxidase (HRP)-conjugated secondary antibodies for $1 \mathrm{~h}$ at $37^{\circ} \mathrm{C}$, followed by enhanced chemiluminescence (Millipore). The signals were captured, and the intensity of the protein bands was quantified using Image J software (NIH, Bethesda, MD, USA) (22). 

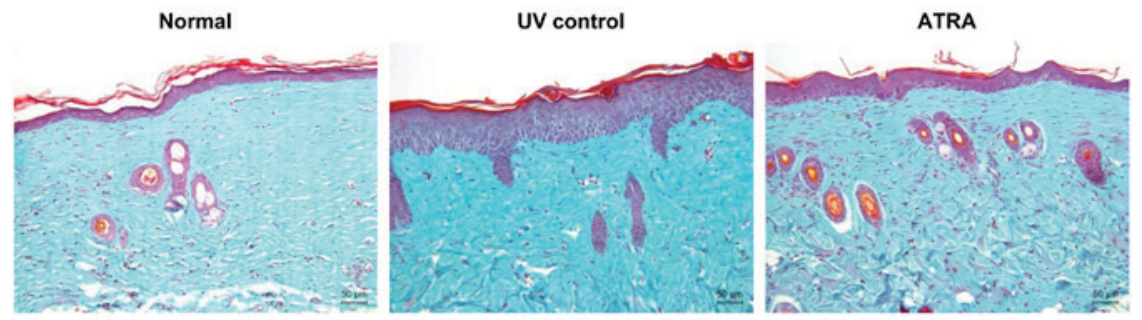

ATRA+RAR antagonist

ATRA+RXR antagonist

RAR agonist
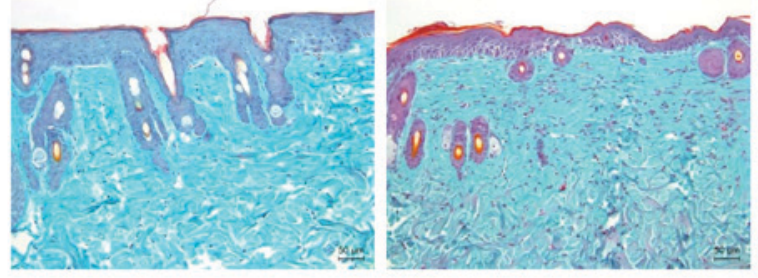

RXR agonist

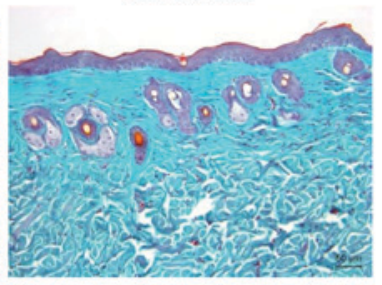

RAR agonist+RAR antagonis

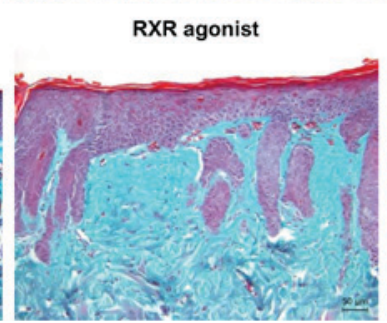

RXR agonist+RXR antagonist
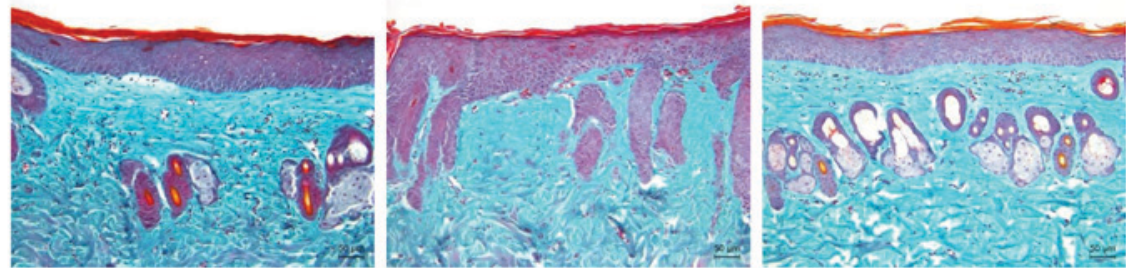

Figure 1. Effects of all-trans retinoic acid (ATRA) and retinoic acid receptor (RAR) and retinoid X receptor (RXR) agonists on the collagen fibers in photoaged mouse skin. Skin tissue sections were stained with Masson's trichrome, and collagen fibers were stained in light green. Original magnification, x200. UV, ultraviolet.

Statistical analysis. All data are presented as the means \pm SD. Statistical analysis was performed using SPSS 19.0 software (SPSS, Inc., Chicago, IL, USA). Data were analyzed using one-way ANOVA or a Student's t-test to perform comparisons between two groups. A P-value of $<0.05$ was considered to indicate a statistically significant difference.

\section{Results}

Histological analysis. Histological sections of the dorsal skin were subjected to Masson's trichrome staining to visualize the changes in collagen fibers in the dermis. As shown in Fig. 1, the normal group displayed regularly arranged collagen fibers. Compared with the normal group, UV irradiation caused large amounts of abnormal, fragmented and disorganized collagen fibers in the UV control group. ATRA and RAR agonist improved the UV-induced damage to collagen fibers, whereas these effects were markedly inhibited by RAR antagonist. Similar changes in collagen fibers were observed either between the ATRA and ATRA + RXR antagonist groups or between the RXR agonist and UV control groups. These results indicated that ATRA and RAR agonist could ameliorate the UV-induced damage to skin collagen fibers through RAR.

Estimation of collagen content. The collagen content was determined through measuring the amount of Hyp. As shown in Fig. 2, UV irradiation induced a $34.86 \%$ decrease in the collagen content ( $\mathrm{P}<0.01$, vs. normal group). ATRA and RAR agonist significantly increased the collagen content by 29.83 and $25.00 \%$, respectively, as compared with the UV control group (all $\mathrm{P}<0.01$, vs. UV control group). However, these effects

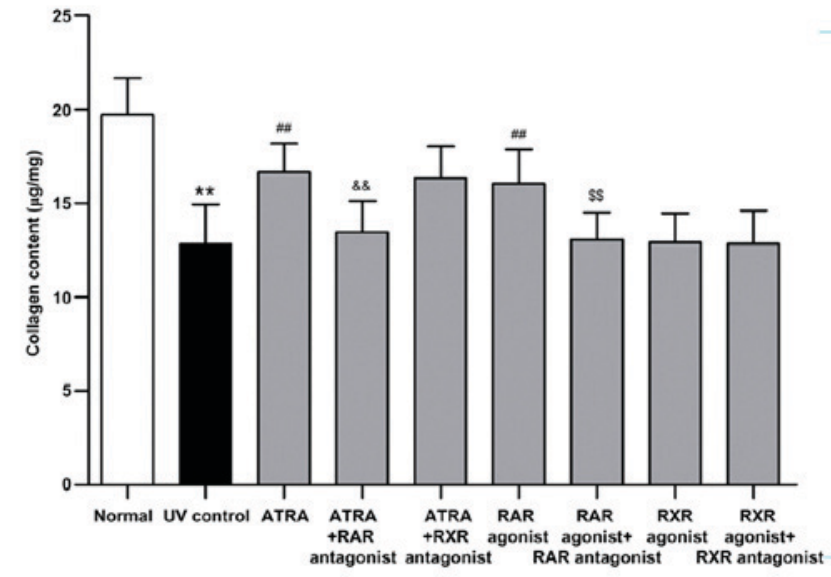

Figure 2. Effects of all-trans retinoic acid (ATRA) and retinoic acid receptor (RAR) and retinoid $\mathrm{X}$ receptor (RXR) agonists on the collagen content in photoaged mouse skin. Data are presented as the means $\pm \mathrm{SD}(\mathrm{n}=8)$. ${ }^{* *} \mathrm{P}<0.01$ vs. normal group; ${ }^{\# \#} \mathrm{P}<0.01$ vs. ultraviolet (UV) control group; ${ }^{\& \&} \mathrm{P}<0.01$ vs. ATRA group; ${ }^{\$ \$} \mathrm{P}<0.01$ vs. RAR agonist group.

were significantly suppressed by RAR antagonist (all $\mathrm{P}<0.01$, vs. ATRA and RAR agonist groups, respectively). There was no significant difference in the collagen content either between the ATRA and ATRA + RXR antagonist groups or between the RXR agonist and UV control groups. These results were consistent with those of Masson's trichrome staining.

Effects of ATRA on the protein expression of MMPs and type I procollagen in photoaged skin. UV irradiation stimulates collagen breakdown and inhibits procollagen synthesis (1). 
A

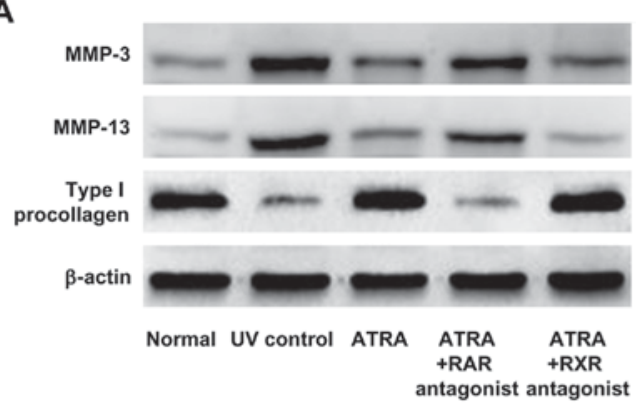

C

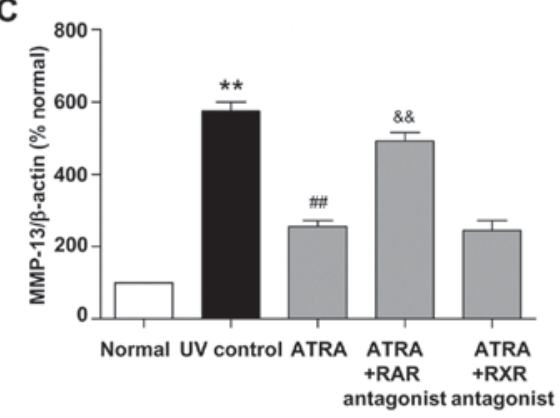

B

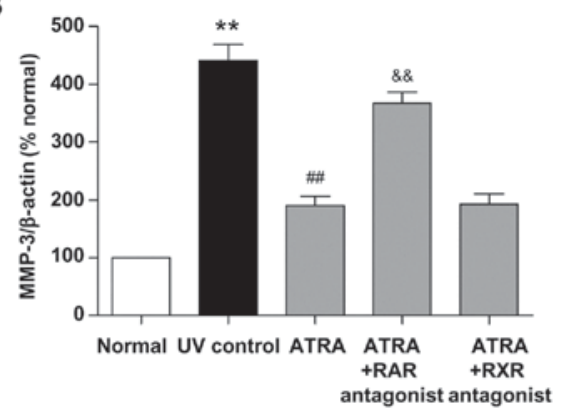

D

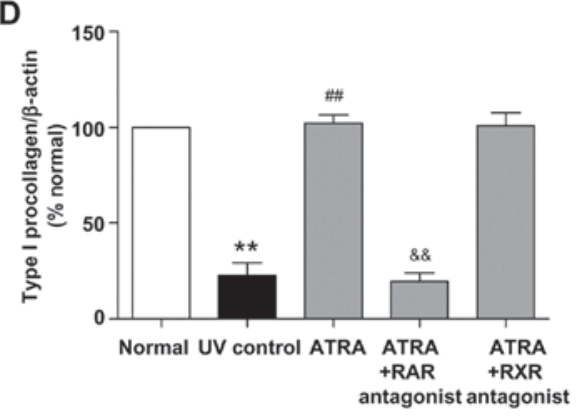

Figure 3. Effects of all-trans retinoic acid (ATRA) on the protein expression of matrix metalloproteinase (MMP)-3, MMP-13 and type I procollagen in photoaged mouse skin. (A) Representative western blots of MMP-3, MMP-13, type I procollagen and $\beta$-actin protein expression. (B-D) Quantification of MMP-3, MMP-13 and type I procollagen protein levels (normalized to $\beta$-actin). Data are presented as the means $\pm \mathrm{SD}(\mathrm{n}=8)$. ${ }^{* *} \mathrm{P}<0.01$ vs. normal group; ${ }^{\sharp \#} \mathrm{P}<0.01 \mathrm{vs}$. ultraviolet (UV) control group; \&\& $<<0.01$ vs. ATRA group. RAR, retinoic acid receptor; RXR, retinoid X receptor.

MMP-3 and MMP-13 are the key regulators of collagen degradation in photoaged mouse skin. To investigate the mechanism underlying the beneficial histological effects of ATRA on photoaged skin, the protein expression of MMP-3,MMP-13 and type I procollagen was detected by western blotting. As shown in Fig. 3, UV irradiation significantly increased the protein levels of MMP-3 and MMP-13 and decreased the protein level of type I procollagen (all $\mathrm{P}<0.01$, vs. normal group). ATRA significantly reduced MMP-3 and MMP-13 protein levels and increased type I procollagen protein level (all $\mathrm{P}<0.01$, vs. UV control group), while these effects were markedly inhibited by RAR antagonist (all $\mathrm{P}<0.01$, vs. ATRA group). There was no significant difference in the protein levels of MMP-3, MMP-13 and type I procollagen between the ATRA and ATRA + RXR antagonist groups. These data indicated that ATRA could stimulate the protein expression of type I procollagen and inhibit the protein expression of MMP-3 and MMP-13 through RAR in photoaged skin.

Effects of RAR and RXR agonists on the protein expression of MMPs and type I procollagen in photoaged skin. We also examined the effects of RAR and RXR agonists on the protein expression of MMP-3, MMP-13 and type I procollagen in photoaged skin. As shown in Figs. 4 and 5, UV irradiation resulted in a significant increase in the protein levels of MMP-3 and MMP-13 and a significant decrease in the protein level of type I procollagen (all $\mathrm{P}<0.01$, vs. normal group). RAR agonist prominently reduced MMP-3 and MMP- 13 protein levels and increased type I procollagen protein level (all $\mathrm{P}<0.01$, vs. UV control group), whereas these effects were significantly suppressed by RAR antagonist (all $\mathrm{P}<0.01$, vs. RAR agonist group). There was no significant difference in the protein levels of MMP-3, MMP-13 and type I procollagen between the RXR agonist and UV control groups. These data demonstrated that RAR agonist rather than RXR agonist could stimulate the protein expression of type I procollagen and inhibit the protein expression of MMP-3 and MMP-13 through RAR in photoaged skin.

Effects of ATRA on the protein expression of c-Jun and c-Fos in photoaged skin. Transcription factor AP-1 is mainly composed of c-Jun and c-Fos proteins and plays an important role in the mechanism of photoaging (3). To investigate the effect of ATRA on AP-1 expression in photoaged skin, the protein expression of c-Jun and c-Fos was detected by western blotting. As shown in Fig. 6, UV irradiation significantly increased the protein level of c-Jun ( $\mathrm{P}<0.01$, vs. normal group). ATRA significantly reduced c-Jun protein level $(\mathrm{P}<0.01$, vs. UV control group), while this effect was markedly inhibited by RAR antagonist $(\mathrm{P}<0.01$, vs. ATRA group). There was no significant difference in the protein level of c-Jun between the ATRA and ATRA + RXR antagonist groups. Moreover, no significant difference in the c-Fos protein level was observed between all groups. These results indicated that ATRA could down-regulate the protein expression of c-Jun through RAR in photoaged skin.

Effects of RAR and RXR agonists on the protein expression of c-Jun and c-Fos in photoaged skin. We also examined the effects of RAR and RXR agonists on the protein expression of c-Jun and c-Fos in photoaged skin. As shown in Figs. 7 and 8, UV irradiation resulted in a significant increase in the protein level of c-Jun ( $\mathrm{P}<0.01$, vs. normal group). RAR agonist prominently reduced c-Jun protein level $(\mathrm{P}<0.01$, vs. UV control group), whereas this effect was significantly suppressed by 
A

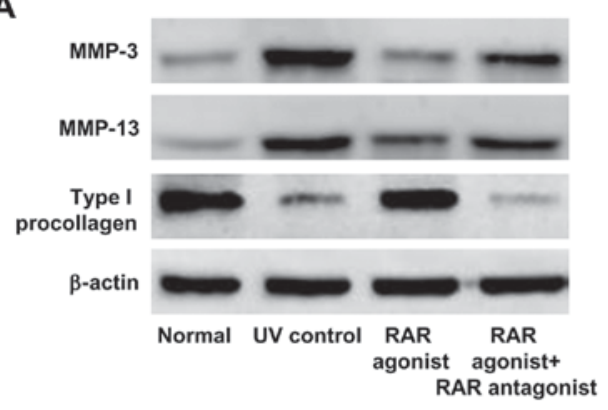

C

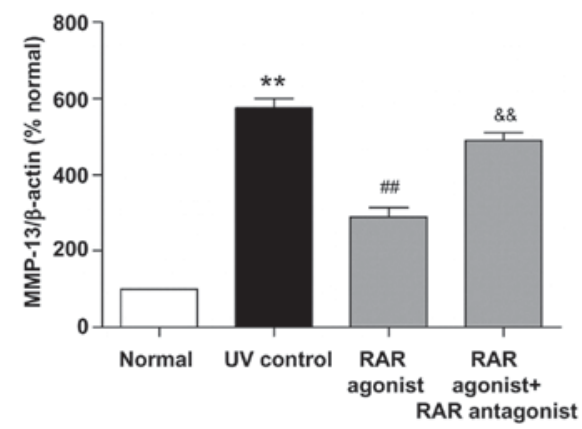

B

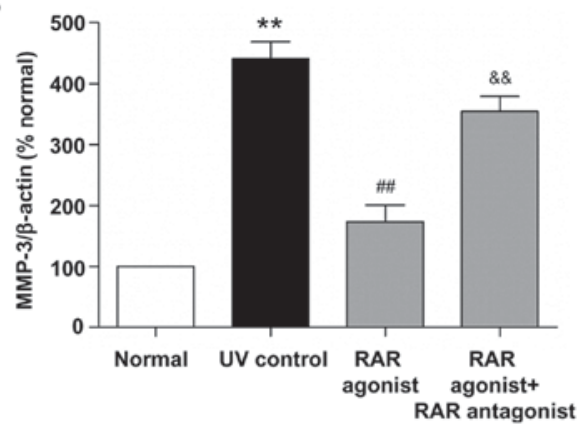

D

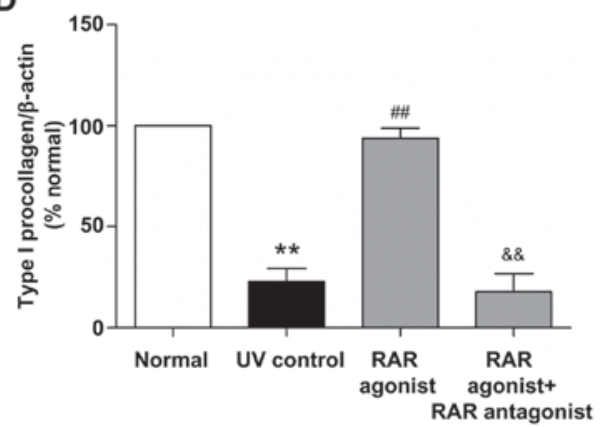

Figure 4. Effects of retinoic acid receptor (RAR) agonist on the protein expression of matrix metalloproteinase (MMP)-3, MMP-13 and type I procollagen in photoaged mouse skin. (A) Representative western blots of MMP-3, MMP-13, type I procollagen and $\beta$-actin protein expression. (B-D) Quantification of MMP-3, MMP-13 and type I procollagen protein levels (normalized to $\beta$-actin). Data are presented as the means $\pm \mathrm{SD}(\mathrm{n}=8)$. ${ }^{* *} \mathrm{P}<0.01$ vs. normal group; ${ }^{\# \#} \mathrm{P}<0.01$ vs. ultraviolet (UV) control group; ${ }^{\& \&} \mathrm{P}<0.01$ vs. RAR agonist group.

RAR antagonist ( $\mathrm{P}<0.01$, vs. RAR agonist group). There was no significant difference in the protein level of c-Jun between the RXR agonist and UV control groups. Furthermore, RAR and RXR agonists had no significant effect on the c-Fos protein level in photoaged skin. These results demonstrated that RAR agonist rather than RXR agonist could down-regulate the protein expression of c-Jun through RAR in photoaged skin.

\section{Discussion}

Chronic exposure to UV irradiation disrupts the normal architecture of skin connective tissue, impairs skin function and ultimately causes photoaging (1). RA, which is the bioactive metabolite of vitamin A, has demonstrated efficacy in the treatment of photoaged skin (12-14). However, the mechanism of its action in the treatment of photoaged skin remains unclear. Thus, in the present study, we applied ATRA, RAR and RXR agonists, as well as RAR and RXR antagonists to the photoaged mouse skin, explored whether the therapeutic effects of RA on photoaged skin are mediated by RAR and/or RXR in mice and investigated the underlying mechanism.

Disorganization of collagen fibers and reduction of collagen content are the prominent features of photoaged skin $(1,3)$. In this study, Masson's trichrome staining and Hyp assay were used to evaluate the effects of ATRA and RAR and RXR agonists on the collagen fibers and collagen content in photoaged skin. Our results showed that UV irradiation caused the fragmented and disorganized collagen fibers and the decreased collagen content, which was consistent with previous studies $(20,23,24)$. ATRA and RAR agonist not only improved the UV-induced damage to collagen fibers but also increased the collagen content in photoaged skin. However, these beneficial effects were markedly inhibited by RAR antagonist. RXR agonist had no significant effect on the collagen fibers and collagen content in photoaged skin (Figs. 1 and 2). Therefore, ATRA and RAR agonist could ameliorate the UV-induced damage to skin collagen fibers and increase the collagen content in photoaged skin through RAR.

UV irradiation causes alterations of dermal collagen through stimulating collagen breakdown and inhibiting procollagen synthesis (1). MMP-3 and MMP-13 are primarily responsible for the degradation of collagen in photoaged mouse skin (5-7). Type I procollagen can be converted into the major structural protein in dermal ECM. Thus, the regulation of MMP-3, MMP-13 and type I procollagen may be an effective strategy for the treatment of photoaged skin in mice. In the present study, UV irradiation significantly increased the protein levels of MMP-3 and MMP-13 and decreased the protein level of type I procollagen. ATRA and RAR agonist significantly reduced MMP-3 and MMP-13 protein levels and increased type I procollagen protein level compared with the UV control group, whereas these effects were markedly inhibited by RAR antagonist (Figs. 3 and 4). RXR agonist had no significant effect on the protein levels of MMP-3, MMP-13 and type I procollagen in photoaged skin (Fig. 5). Therefore, ATRA and RAR agonist could stimulate the protein expression of type I procollagen and inhibit the protein expression of MMP-3 and MMP-13 through RAR in photoaged skin.

UV-induced transcription factor AP-1, which is composed of elevated c-Jun and constitutively expressed c-Fos, increases MMPs transcription and decreases procollagen synthesis in the process of photoaging $(3,25,26)$. RA can antagonize UV 
A

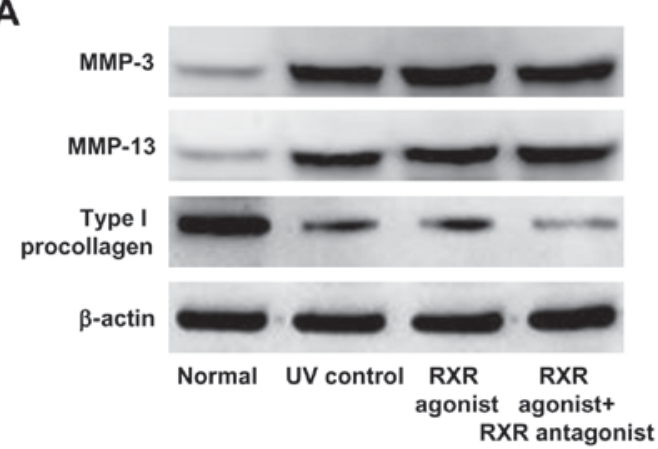

B

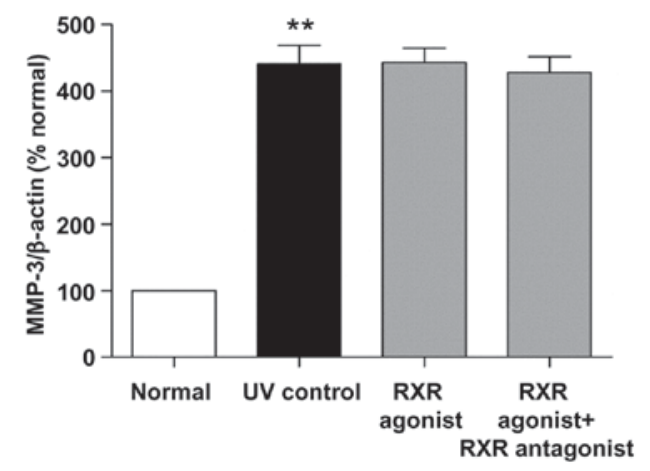

D

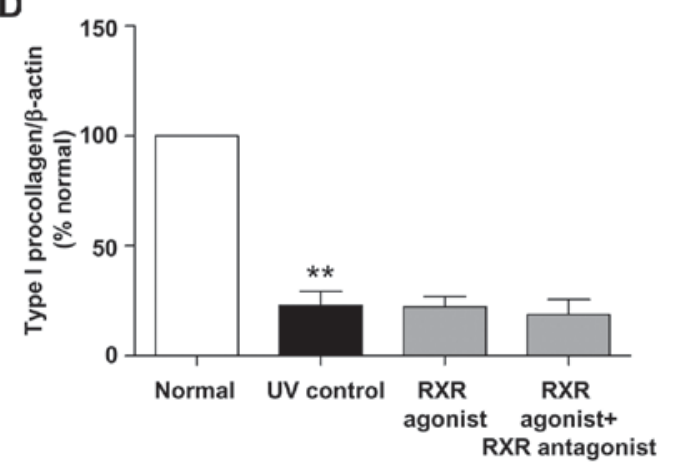

Figure 5. Effects of retinoid X receptor (RXR) agonist on the protein expression of matrix metalloproteinase (MMP)-3, MMP-13 and type I procollagen in photoaged mouse skin. (A) Representative western blots of MMP-3, MMP-13, type I procollagen and $\beta$-actin protein expression. (B-D) Quantification of MMP-3, MMP-13 and type I procollagen protein levels (normalized to $\beta$-actin). Data are presented as the means $\pm \mathrm{SD}(\mathrm{n}=8)$. "* $\mathrm{P}<0.01 \mathrm{vs.} \mathrm{normal} \mathrm{group.} \mathrm{UV,}$ ultraviolet.
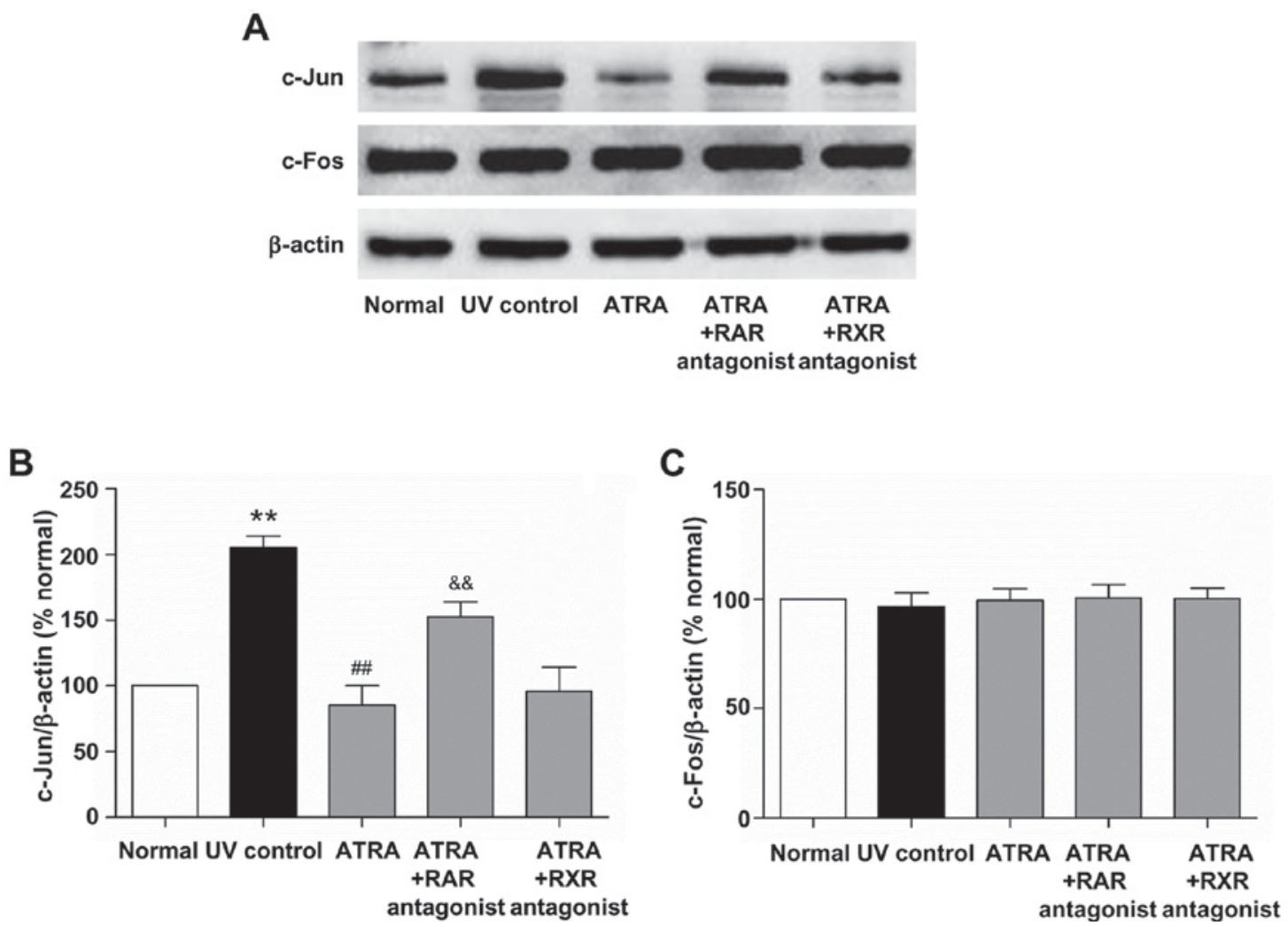

Figure 6. Effects of all-trans retinoic acid (ATRA) on the protein expression of c-Jun and c-Fos in photoaged mouse skin. (A) Representative western blots of c-Jun, c-Fos and $\beta$-actin protein expression. (B and C) Quantification of c-Jun and c-Fos protein levels (normalized to $\beta$-actin). Data are presented as the means $\pm \mathrm{SD}(\mathrm{n}=8) .{ }^{* *} \mathrm{P}<0.01$ vs. normal group; ${ }^{\# \#} \mathrm{P}<0.01$ vs. ultraviolet (UV) control group; ${ }^{\& \&} \mathrm{P}<0.01$ vs. ATRA group. RAR, retinoic acid receptor; RXR, retinoid $\mathrm{X}$ receptor. 

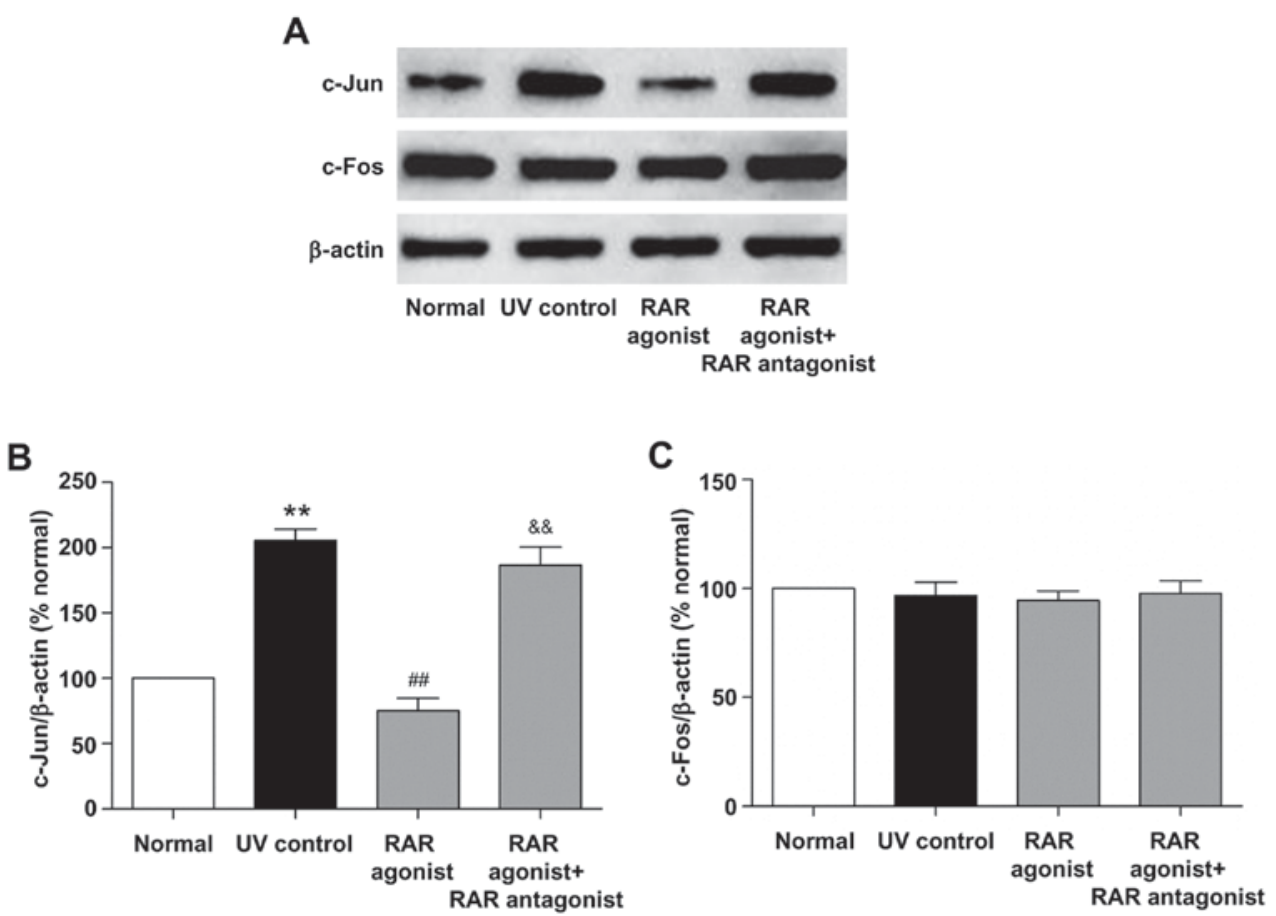

Figure 7. Effects of retinoic acid receptor (RAR) agonist on the protein expression of c-Jun and c-Fos in photoaged mouse skin. (A) Representative western blots of c-Jun, c-Fos and $\beta$-actin protein expression. (B and C) Quantification of c-Jun and c-Fos protein levels (normalized to $\beta$-actin). Data are presented as the means $\pm \mathrm{SD}(\mathrm{n}=8) .{ }^{* *} \mathrm{P}<0.01$ vs. normal group; ${ }^{\# \#} \mathrm{P}<0.01$ vs. ultraviolet (UV) control group; ${ }^{\&}{ }^{\&} \mathrm{P}<0.01$ vs. RAR agonist group.
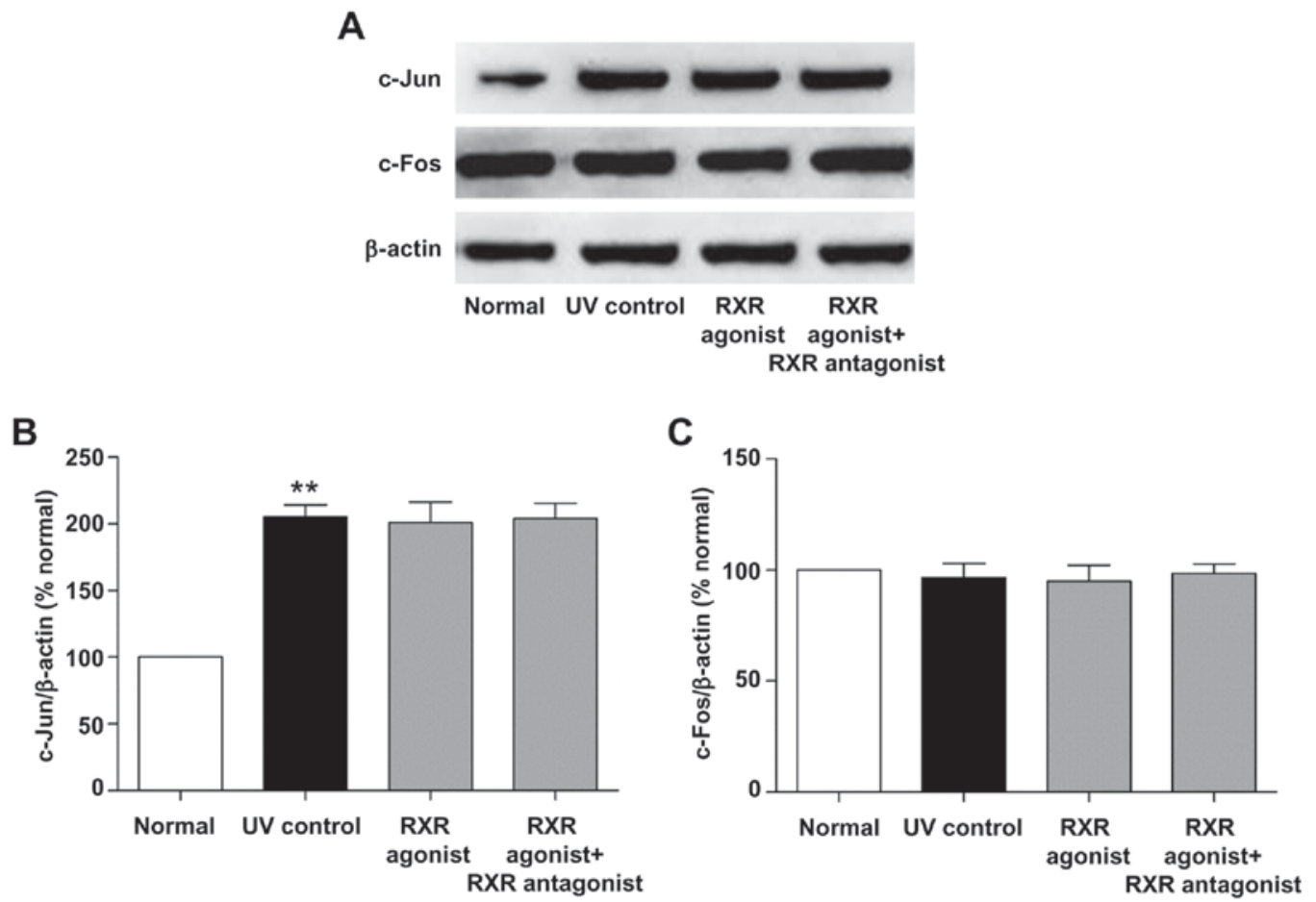

Figure 8. Effects of retinoid X receptor (RXR) agonist on the protein expression of c-Jun and c-Fos in photoaged mouse skin. (A) Representative western blots of c-Jun, c-Fos and $\beta$-actin protein expression. (B and C) Quantification of c-Jun and c-Fos protein levels (normalized to $\beta$-actin). Data are presented as the means $\pm \mathrm{SD}(\mathrm{n}=8) .{ }^{* *} \mathrm{P}<0.01$ vs. normal group. $\mathrm{UV}$, ultraviolet.

activation of AP-1 by inhibiting the induction of c-Jun protein in human skin (26). In this study, we further investigated whether RA stimulated type I procollagen protein expression and inhibited MMP-3 and MMP-13 protein expression through
AP-1 pathway in photoaged skin. Our results demonstrated that ATRA and RAR agonist significantly reduced the protein level of c-Jun in photoaged skin, while these effects were markedly inhibited by RAR antagonist (Figs. 6 and 7). There was 
no significant difference in the protein level of c-Jun between the RXR agonist and UV control groups (Fig. 8). In addition, ATRA and RAR and RXR agonists had no significant effect on the c-Fos protein level in photoaged skin (Figs. 6-8). These findings indicated that ATRA and RAR agonist could stimulate type I procollagen protein expression and inhibit MMP-3 and MMP-13 protein expression by down-regulating c-Jun protein expression in photoaged skin, which was mediated by RAR.

In conclusion, our study indicates for the first time that ATRA and RAR agonist could ameliorate the UV-induced damage to skin collagen fibers and increase the collagen content in photoaged skin through RAR. In addition, ATRA and RAR agonist could stimulate type I procollagen protein expression and inhibit MMP-3 and MMP-13 protein expression by down-regulating c-Jun protein expression in photoaged skin, which was mediated by RAR. Taken together, these findings suggest that RA may ameliorate photoaged skin through RAR-mediated pathway in mice, providing a theoretical basis for clinical treatment of photoaged skin.

\section{Acknowledgements}

The present study was supported by the National Natural Science Foundation of China (no. 81271741).

\section{References}

1. Quan T, Qin Z, Xia W, Shao Y, Voorhees JJ and Fisher GJ: Matrix-degrading metalloproteinases in photoaging. J Investig Dermatol Symp Proc 14: 20-24, 2009.

2. Svobodová A and Vostálová J: Solar radiation induced skin damage: Review of protective and preventive options. Int J Radiat Biol 86: 999-1030, 2010.

3. Yaar M and Gilchrest BA: Photoageing: Mechanism, prevention and therapy. Br J Dermatol 157: 874-887, 2007.

4. Quan T, He T, Voorhees JJ and Fisher GJ: Ultraviolet irradiation induces Smad7 via induction of transcription factor AP-1 in human skin fibroblasts. J Biol Chem 280: 8079-8085, 2005.

5. Nelson AR, Fingleton B, Rothenberg ML and Matrisian LM: Matrix metalloproteinases: Biologic activity and clinical implications. J Clin Oncol 18: 1135-1149, 2000.

6. Feng XX, Yu XT, Li WJ, Kong SZ, Liu YH, Zhang X, Xian YF, Zhang XJ, Su ZR and Lin ZX: Effects of topical application of patchouli alcohol on the UV-induced skin photoaging in mice. Eur J Pharm Sci 63: 113-123, 2014.

7. Schorpp M, Mattei MG, Herr I, Gack S, Schaper J and Angel P. Structural organization and chromosomal localization of the mouse collagenase type I gene. Biochem J 308: 211-217, 1995.

8. Ascenso A, Ribeiro H, Marques HC, Oliveira H, Santos C and Simões S: Is tretinoin still a key agent for photoaging management? Mini Rev Med Chem 14: 629-641, 2014.

9. Torok HM and Pillai R: Safety and efficacy of micronized tretinoin gel $(0.05 \%)$ in treating adolescent acne. J Drugs Dermatol 10: 647-652, 2011.

10. Weinstein GD, Koo JY, Krueger GG, Lebwohl MG, Lowe NJ, Menter MA, Lew-Kaya DA, Sefton J, Gibson JR and Walker PS; Tazarotene Cream Clinical Study Group: Tazarotene cream in the treatment of psoriasis: Two multicenter, double-blind, randomized, vehicle-controlled studies of the safety and efficacy of tazarotene creams $0.05 \%$ and $0.1 \%$ applied once daily for 12 weeks. J Am Acad Dermatol 48: 760-767, 2003.
11. Lippman SM, Parkinson DR, Itri LM, Weber RS, Schantz SP, Ota DM, Schusterman MA, Krakoff IH, Gutterman JU and Hong WK: 13-cis-retinoic acid and interferon alpha-2a: Effective combination therapy for advanced squamous cell carcinoma of the skin. J Natl Cancer Inst 84: 235-241, 1992.

12. Ho ET, Trookman NS, Sperber BR, Rizer RL, Spindler R, Sonti S, Gotz V and Mehta R: A randomized, double-blind, controlled comparative trial of the anti-aging properties of non-prescription tri-retinol $1.1 \%$ vs. prescription tretinoin $0.025 \%$. J Drugs Dermatol 11: 64-69, 2012.

13. Bouloc A, Vergnanini AL and Issa MC: A double-blind randomized study comparing the association of Retinol and LR2412 with tretinoin $0.025 \%$ in photoaged skin. J Cosmet Dermatol 14: 40-46, 2015.

14. Bagatin E, Guadanhim LR, Enokihara MM, Sanudo A, Talarico S, Miot HA and Gibson L: Low-dose oral isotretinoin versus topical retinoic acid for photoaging: A randomized, comparative study. Int J Dermatol 53: 114-122, 2014.

15. Konta T, Xu Q, Furusu A, Nakayama K and Kitamura M: Selective roles of retinoic acid receptor and retinoid $X$ receptor in the suppression of apoptosis by all-trans-retinoic acid. J Biol Chem 276: 12697-12701, 2001

16. Mihály J, Gericke J, Lucas R, de Lera AR, Alvarez S, Törőcsik D and Rühl R: TSLP expression in the skin is mediated via RAR $\gamma$-RXR pathways. Immunobiology 221: 161-165, 2016.

17. Gericke J, Ittensohn J, Mihály J, Alvarez S, Alvarez R, Töröcsik D, de Lera AR and Rühl R: Regulation of retinoid-mediated signaling involved in skin homeostasis by RAR and RXR agonists/antagonists in mouse skin. PLoS One 8: e62643, 2013.

18. Mukherjee S, Date A, Patravale V, Korting HC, Roeder A and Weindl G: Retinoids in the treatment of skin aging: An overview of clinical efficacy and safety. Clin Interv Aging 1: 327-348, 2006.

19. Kang S, Bergfeld W, Gottlieb AB, Hickman J, Humeniuk J, Kempers S, Lebwohl M, Lowe N, McMichael A, Milbauer J, et al: Long-term efficacy and safety of tretinoin emollient cream $0.05 \%$ in the treatment of photodamaged facial skin: A two-year, randomized, placebo-controlled trial. Am J Clin Dermatol 6: 245-253, 2005.

20. Wang XF, Huang YF, Wang L, Xu LQ, Yu XT, Liu YH, Li CL, Zhan JY, Su ZR, Chen JN and Zeng HF: Photo-protective activity of pogostone against UV-induced skin premature aging in mice. Exp Gerontol 77: 76-86, 2016.

21. Neuman RE and Logan MA: The determination of collagen and elastin in tissues. J Biol Chem 186: 549-556, 1950.

22. Huang $X$, Zhu $B$, Wang $X$, Xiao $R$ and Wang $C$ : Three-dimensional co-culture of mesenchymal stromal cells and differentiated osteoblasts on human bio-derived bone scaffolds supports active multi-lineage hematopoiesis in vitro: Functional implication of the biomimetic HSC niche. Int J Mol Med 38: 1141-1151, 2016.

23. Kong SZ, Chen HM, Yu XT, Zhang X, Feng XX, Kang XH, Li WJ, Huang N, Luo H and Su ZR: The protective effect of $18 \beta$-Glycyrrhetinic acid against UV irradiation induced photoaging in mice. Exp Gerontol 61: 147-155, 2015.

24. Hwang E, Park SY, Lee HJ, Lee TY, Sun ZW and Yi TH: Gallic acid regulates skin photoaging in UVB-exposed fibroblast and hairless mice. Phytother Res 28: 1778-1788, 2014.

25. Hwang KA, Yi BR and Choi KC: Molecular mechanisms and in vivo mouse models of skin aging associated with dermal matrix alterations. Lab Anim Res 27: 1-8, 2011.

26. Fisher GJ, Talwar HS, Lin J, Lin P, McPhillips F, Wang Z, Li X, Wan Y, Kang S and Voorhees JJ: Retinoic acid inhibits induction of c-Jun protein by ultraviolet radiation that occurs subsequent to activation of mitogen-activated protein kinase pathways in human skin in vivo. J Clin Invest 101: 1432-1440, 1998. 